\title{
Molecular Epidemiology of Koi Herpesvirus
}

\author{
Jun Kurita ${ }^{*}$, Kei Yuasa ${ }^{2}$, Takafumi Ito ${ }^{1}$, Motohiko Sano ${ }^{2}$, Ronald P. Hedrick ${ }^{3}$, \\ Marc Y. Engelsma ${ }^{4}$, Olga L. M. Haenen ${ }^{4}$, Agus Sunarto ${ }^{5}$, Edy Barkat Kholidin ${ }^{6}$, \\ Hsin-Yiu Chou ${ }^{7}$, Ming-Chen Tung ${ }^{8}$, Leobert de la Peña ${ }^{9}$, Gilda Lio-Po ${ }^{9}$, \\ Chien $\mathrm{Tu}^{10}$, Keith Way ${ }^{11}$ and Takaji lida ${ }^{2}$ \\ ${ }^{1}$ Tamaki Station, National Research Institute of Aquaculture, Fisheries Research Agency, Mie 519-0423, Japan \\ ${ }^{2}$ Nansei Station, National Research Institute of Aquaculture, Fisheries Research Agency, Mie 516-0193, Japan \\ ${ }^{3}$ Department of Medicine and Epidemiology, School of Veterinary Medicine, University of California, Davis, \\ California 95616, USA \\ ${ }^{4}$ CIDC-Lelystad, Wageningen UR, Fish and Shellfish Diseases Laboratory, P.O. Box 2004, \\ 8203 AA Lelystad, the Netherlands \\ ${ }^{5}$ Fish Health Research Laboratory, Agency for Marine and Fisheries Research, Jakarta, Indonesia \\ ${ }^{6} J a m b i$ Freshwater Aquaculture Development Center; Sungai Gelam, Kumpeh Ulu, \\ Muara Jambi, Indonesia \\ ${ }^{7}$ Department of Aquaculture, National Taiwan Ocean University, Taiwan, Republic of China \\ ${ }^{8}$ Department of Veterinary Medicine, National Pingtung University of Science \& Technology, \\ Taiwan, Republic of China \\ ${ }^{9}$ Fish Health Section, Southeast Asian Fisheries Development Center Aquaculture Department (SEAFDEC/AQD), \\ Tigbauan, Iloilo 5021, Philippines \\ ${ }^{10}$ Fish Health Laboratory, Animal Health Research Institute, 376 Chung-Cheng Rd Tanshui, 251, \\ Taiwan, Republic of China \\ ${ }^{11}$ Centre for Environment Fisheries and Aquaculture Science (Cefas), Weymouth, \\ Dorset DT4 8UB, UK
}

(Received March 12, 2008)

\begin{abstract}
Three regions of koi herpesvirus (KHV) genomic DNA were compared for 34 samples from Japan, six from Indonesia, two from Taiwan, one from the Philippines, 13 from the Netherlands, one from the UK, one from the USA and one from Israel. The analyzed genomic regions included known PCRdetection targets (Sphl-5, 9/5 and the thymidine kinase gene). The KHVs from Asian countries were very homogeneous, although two variants were noted based on a single nucleotide polymorphism. In contrast, seven variants were found in KHVs from outside of Asia, and although closely related to one another, they were clearly distinct from those from Asian. The results suggest that a clear genetic distinction exists between Asian and European (including each single isolate from the USA and Israel) types of KHV, and that unique types of KHV were independently introduced or emerged in the respective geographic locations.
\end{abstract}

Key words: koi herpesvirus, KHV, Cyprinid herpesvirus-3, CyHV-3, molecular epidemiology, fish herpesvirus, Cyprinus carpio

Koi herpesvirus (KHV), the third cyprinid herpesvirus (CyHV-3) (Waltzek et al., 2005), is highly contagious and can cause disease and mortality in common carp Cyprinus carpio and its ornamental variety, koi. The virus was first isolated from fish cultured in Israel and USA, respectively, in 1998 (Hedrick et al., 2000). Subsequently, KHV was also isolated in Germany (Neukirch and Kunz, 2001; Neukirch et al., 2003*1), Israel

\footnotetext{
* Corresponding author

E-mail: kuritaj@fra.affrc.go.jp

*1 Neukirch, M. and D. Steinhagen (2003): Influence of temperature and $\mathrm{pH}$ on the infectivity of viruses isolated from koi.
}

(Perelberg et al., 2003; Ronen et al., 2003) and the UK (Way et al., 2001*2). The disease associated with KHV had occurred earlier in Germany in 1997-1998 (Bretzinger et al., 1999) and the presence of this viral

\footnotetext{
Abstract of the 11th International Conference of the European Association of Fish Pathologists-in Malta.

*2 Way, Keith, R.-M. Le Deuff, L. Ecclestone, S. W. Feist, P. F. Dixon, W. H. Wildgoose and R. P. Hedrick (2001): Isolation of a herpesvirus during disease outbreaks in adult koi carp, Cyprinus carpio, in the UK. Abstract of the 10th International Conference of the European Association of Fish Pathologistsin Dublin.
} 
Table 1. List of Japanese KHV samples

\begin{tabular}{|c|c|c|c|c|c|c|}
\hline $\begin{array}{c}\text { Sample } \\
\text { No. }\end{array}$ & Prefecture & Place of sampling & Fish & Farmed or wild & Year & $\begin{array}{l}\text { Introduction of common } \\
\text { carp from Lake } \\
\text { Kasumigaura }\end{array}$ \\
\hline $1^{* 1}$ & Okayama & river & common carp & wild & 2003 & no \\
\hline $2^{* 1}$ & Okayama & koi farm & koi & farmed & 2003 & no \\
\hline $3^{* 1}$ & Okayama & lake & common carp & wild & 2003 & no \\
\hline $4^{* 1}$ & Okayama & river & koi & wild & 2003 & no \\
\hline $5^{* 2}$ & Ibaraki & Lake Kasumigaura & common carp & farmed & 2003 & - \\
\hline 6 & Ibaraki & Lake Kitaura & common carp & farmed & 2003 & yes \\
\hline 7 & Miyazaki & fish farm & common carp & farmed & 2003 & yes \\
\hline 8 & Miyazaki & restaurant & common carp & farmed & 2003 & yes \\
\hline 9 & Yamanashi & fishing pond ${ }^{* 3}$ & common carp & farmed & 2003 & yes \\
\hline 10 & Mie & fishing pond & common carp & farmed & 2003 & yes \\
\hline 11 & Aomori & fish farm & koi/common carp & farmed & 2003 & yes \\
\hline 12 & Fukuoka & unknown & common carp & unknown & 2003 & yes \\
\hline 13 & Fukuoka & unknown & common carp & unknown & 2003 & yes \\
\hline 14 & Saitama & fishing pond & common carp & farmed & 2003 & yes \\
\hline 15 & Shiga & fish farm & common carp & farmed & 2003 & yes \\
\hline 16 & Osaka & river & common carp & wild & 2003 & no \\
\hline 17 & Niigata & fishing pond & common carp & farmed & 2003 & unknown \\
\hline 18 & Gifu & fishing pond & common carp & unknown & 2003 & unknown \\
\hline 19 & Chiba & fishing pond & common carp & farmed & 2003 & yes \\
\hline 20 & Kagoshima & river & koi & wild & 2003 & no \\
\hline 21 & Kagoshima & river & koi & wild & 2003 & no \\
\hline 22 & Kyoto & river & common carp & wild & 2003 & unknown \\
\hline 23 & Miyazaki & restaurant & common carp & farmed & 2003 & unknown \\
\hline 24 & Tokyo & fishing pond & unknown & farmed & 2003 & yes \\
\hline 25 & Tottori & private household & koi & farmed & 2003 & no \\
\hline 26 & Kyoto & unknown & unknown & wild & 2003 & unknown \\
\hline 27 & Kyoto & unknown & unknown & wild & 2003 & unknown \\
\hline 28 & Kyoto & unknown & unknown & unknown & 2003 & unknown \\
\hline 29 & Shizuoka & fishing pond & common carp & farmed & 2003 & yes \\
\hline 30 & Gifu & unknown & unknown & unknown & 2003 & unknown \\
\hline 31 & Nara & reservoir pond & koi & farmed & 2003 & no \\
\hline 32 & Miyazaki & unknown & common carp & wild & 2003 & unknown \\
\hline 33 & Tottori & private household & koi & unknown & 2003 & unknown \\
\hline 34 & Niigata & unknown & common carp & farmed & 2003 & yes \\
\hline
\end{tabular}

*1 : The outbreak of Lake Kasumigaura was preceded by the outbreaks, which became the sources of these samples.

*2 : KHV NRIA-0301 was isolated from this sample.

$* 3$ : A fishing pond is a pond stocked with carp for fee fishing.

infection was confirmed in other European countries and in South Africa (Haenen et al., 2004) since 2001. Recently, KHV was detected by PCR in stored, formalinfixed tissue from carp sampled in the UK in 1996 (Way et al., $2004^{* 3}$ ). In Indonesia, the first outbreak of KHV occurred in fish imported from China through Hong Kong

\footnotetext{
*3 Way, Keith, N. D. Beevers, C. L. Joiner, C. B Longshaw, S. St-Hilaire, D. M. Stone, K. L. Denham and P. F. Dixon. (2004): Koi herpesvirus in the UK: Detection in archive samples and spread of the virus to wild carp, Abstract of the 6th International Symposium on Viruses of Lower Vertebratesin Hakodate.
}

in 2002 (Yuasa, 2004; Sunarto et al., 2005). In the same year, a mass mortality of carp caused by KHV occurred in Taiwan (Tu et al., 2004).

In Japan, KHV was first detected by PCR from diseased common carp cultured in Lake Kasumigaura in Ibaraki Prefecture in October, 2003 (Sano et al., 2004; lida and Sano, 2005; Sano et al., 2005). However, the detection of KHV from frozen fish samples from a disease outbreak in Okayama Prefecture revealed that KHV was introduced into Japan as early as May 2003 (Sano et al., 2004). Although there has been speculation, no definitive information is available for the source 


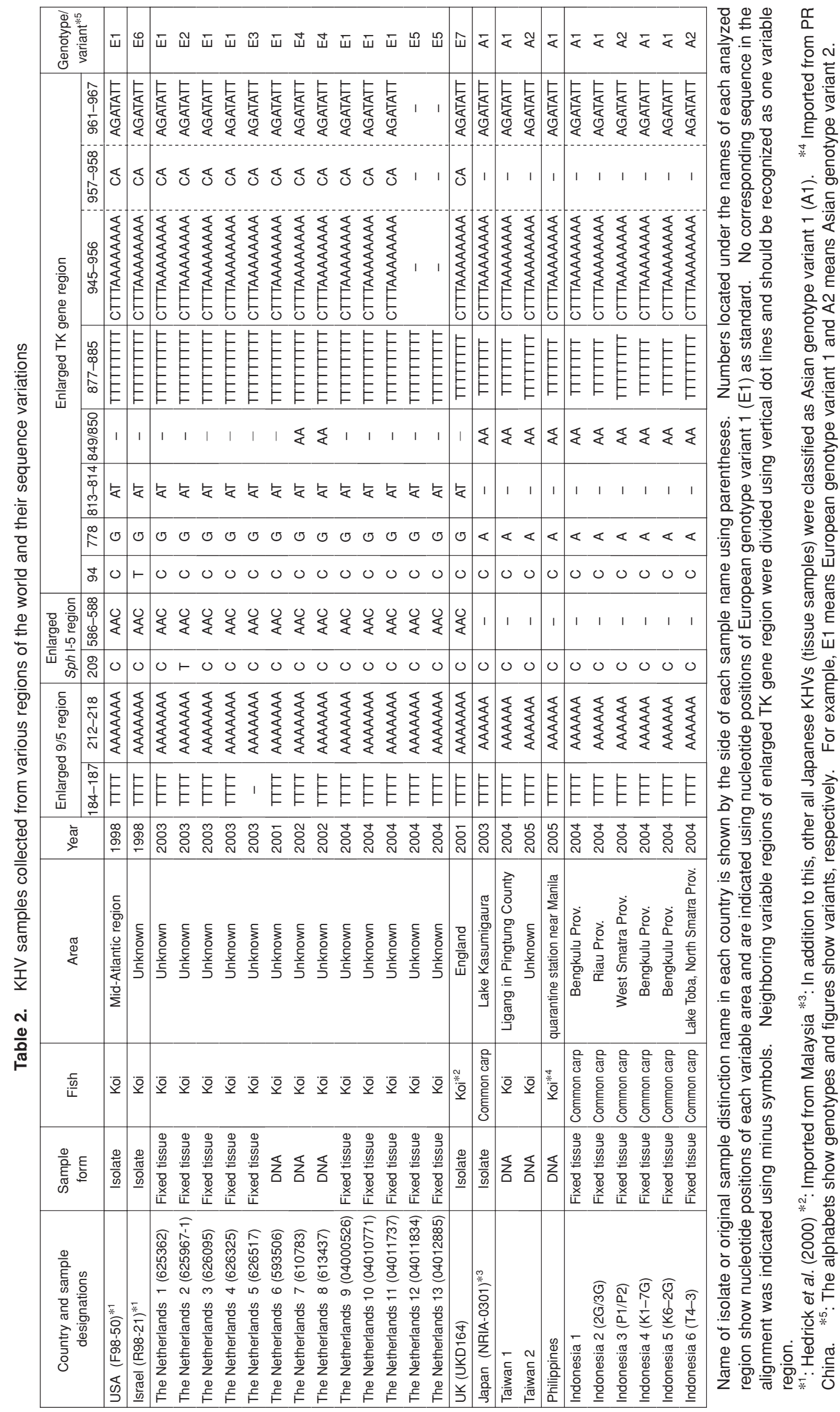


of infection which introduced the virus into Japan. In the present study, we compared regions of the KHV genomic DNA from various countries of the world for sequence variations that would provide insights into the potential routes by which KHV has been spread worldwide.

\section{Materials and Methods}

\section{KHV samples}

Thirty four KHV samples from Japan, six from Indonesia, two from Taiwan, one from the Philippines, 13 from the Netherlands, one from the UK, one from the USA and one from Israel were used for analysis. Japanese samples of KHV were obtained from the tissues (mostly the gills) sent to the National Research Institute of Aquaculture from prefectural governments for the confirmatory diagnosis. In addition, samples from the frozen tissues of dead carp from the outbreaks in Okayama Prefecture (Sano et al., 2004) were also included. The outbreak of Lake Kasumigaura was preceded by these outbreaks.

The information about Japanese and other worldwide samples analyzed in the present study is summarized in Table 1 and Table 2, respectively. The KHV sample from the Philippines was taken from koi transported to a quarantine station near Manila International Airport. The koi were bought during the Aquarama trade show in PR China and imported and confiscated at the airport. KHV samples were collected either as tissues of infected fish, DNA extracts from the tissues, or as isolates of the virus. The samples included common carp and koi, both cultured and wild.

\section{DNA extraction for PCR templates}

For tissue samples, DNA was extracted using the Puregene DNA Purification Kit (Gentra Systems, USA) according to the manufacturer protocol, and the extracted DNA pellet was dissolved in $50 \mu \mathrm{L}$ of nucleasefree distilled water. For KHV isolates, viruses were inoculated onto KF-1 (Hedrick et al., 2000) or CCB cells (Neukirch and Kunz, 2001) and DNA was then prepared from the supernatant of infected cell cultures using Instagene Matrix (Bio-Rad Laboratories, USA).

\section{Primers and PCR amplification for the three genomic regions of $\mathrm{KHV}$}

Primer sequences for amplification of the $610 \mathrm{bp}$ length 'enlarged Sphl-5 region' including the known PCR-detection target of the 290 bp-length 'Sphl-5' region (Gray et al., 2002) are 5'-CGGAGGACCTGATAACCATCA-3' as the forward primer and 5'-GGTAGCTGCAGTCGTGCGAC-3' as the reverse primer. Those for the 611 bp-length 'enlarged 9/5 region' including the known PCR-detection target of the 484 bp-length ' $9 / 5$ ' region (Gilad et al., 2002) are 5'-TCCAGCGACA-
GGATCTCTCG-3' as the forward primer and 5'TCTGCGTGCAGCTCTCCAAC-3' as the reverse primer. Those for the 1,001 bp-length 'enlarged thymidine kinase (TK) gene region' including 651bp-length TK gene open reading frame and nested 409 bp-length PCR-detection target, 'TK' (Bercovier et al., 2005) are 5'-AACGCGGGCCAGCTGAACAT-3' as the forward primer and 5'TGTGTGTATCCCAATAAACG-3' as the reverse primer. Sequence information of upstream and downstream of ' $9 / 5$ ' region was kindly provided by Dr. Gilad (pers. comm.). Other sequence information was obtained by genome walking using demi-specific single primer PCR (Morzunov et al., 1995) or LA-PCR between the region and neighboring genomic fragments. The reaction mixture for PCR contained $2 \mu \mathrm{L}$ of $10 \times$ PCR buffer, $1.6 \mu \mathrm{L}$ of a $2.5 \mathrm{~mm}$ dNTP mixture, $0.5 \mu \mathrm{L}$ of each primer (100 $\mathrm{pmol} / \mu \mathrm{L}$ ), $14.7 \mu \mathrm{L}$ of sterile distilled water, $0.2 \mu \mathrm{L}$ (I U) of Taq DNA polymerase (Ex Taq Hot Start Version; TaKaRa, Japan), and $0.5 \mu \mathrm{L}$ of extracted DNA as template. The thermocycling profile included initial denaturation at $94^{\circ} \mathrm{C}$ for $30 \mathrm{~s}$, followed by 35 cycles of denaturation at $94^{\circ} \mathrm{C}$ for $30 \mathrm{~s}$, annealing at $58^{\circ} \mathrm{C}$ for $30 \mathrm{~s}$, and extension at $72^{\circ} \mathrm{C}$ for $1 \mathrm{~min}$ and a final extension at $72^{\circ} \mathrm{C}$ for $7 \mathrm{~min}$.

\section{Cloning, sequencing and sequence analyses}

The PCR products of expected molecular size by agarose-gel electrophoresis were purified by MinElute PCR Purification Kit (QIAGEN, Germany) and directly sequenced in both orientations with the primers used for amplification or several inner primers (sequences are not shown). When the bands were faint, bands of expected size were collected from the gel and purified by MinElute Gel Extraction Kit (QIAGEN). Then the purified DNAs were ligated into the pCR4-TOPO vector using the TOPO TA Cloning Kit for Sequencing (Invitrogen, USA) and cloned into Escherichia coli strain $\mathrm{DH} 5 \alpha$. Plasmids from at least five independently derived clones were extracted using QIAprep Spin Miniprep Kit (QIAGEN) and sequenced in both orientations and consensus sequences were determined. All sequencing was performed using ABI PRISM BigDye Terminator v.3.1 Cycle Sequencing Kit (Applied Biosystems, USA) and the ABI PRISM 377 DNA sequencer (Applied Biosystems, USA). Alignments of nucleotide and amino acid sequences were basically made using GENETYX-MAC (GENETYX, Japan).

\section{Results and Discussion}

The comparison of the DNA sequences revealed ten variable areas in the three genomic regions of the KHV genome (Table 2). Based on the observed pattern of polymorphism, KHV samples were roughly divided into two groups, designated as Asian and European genotypes with each genotype containing two or seven 


E1-3,6
E5
E7
E4
A1
A2

376

376

376

376

376

376
GAGGTGATGCAGCGTCTGGAGGAATACGACGCCGTGGCCGTCGACGAGGGACAGTTCTTCCCCGACCTCT GAGGTGATGCAGCGTCTGGAGGAATACGACGCCGTGGCCGTCGACGAGGGACAGTTCTTCCCCGACCTCT GAGGTGATGCAGCGTCTGGAGGAATACGACGCCGTGGCCGTCGACGAGGGACAGTTCTTCCCCGACCTCT GAGGTGATGCAGCGTCTGGAGGAATACGACGCCGTGGCCGTCGACGAGGGACAGTTCTTCCCCGACCTCT GAGGTGATGCAGCGTCTGGAGGAATACGACGCCGTGGCCGTCGACGAGGGACAGTTCTTCCCCGACCTCT GAGGTGATGCAGCGTCTGGAGGAATACGACGCCGTGGCCGTCGACGAGGGACAGTTCTTCCCCGACCTCT annealing position of primer $\mathrm{TKcomF}$

E1-3,6 446 ACGAGGGAGTCGTGCAGCTGCTGACCGCGGGCAAGTACGTGATCGTGGCGGCGCTGGACGGGGACTTTAT E5 446 ACGAGGGAGTCGTGCAGCTGCTGACCGCGGGCAAGTACGTGATCGTGGCGGCGCTGGACGGGGACTTTAT E7 446 ACGAGGGAGTCGTGCAGCTGCTGACCGCGGGCAAGTACGTGATCGTGGCGGCGCTGGACGGGGACTTTAT E4 446 ACGAGGGAGTCGTGCAGCTGCTGACCGCGGGCAAGTACGTGATCGTGGCGGCGCTGGACGGGGACTTTAT A1 446 ACGAGGGAGTCGTGCAGCTGCTGACCGCGGGCAAGTACGTGATCGTGGCGGCGCTGGACGGGGACTTTAT

A2 446 ACGAGGGAGTCGTGCAGCTGCTGACCGCGGGCAAGTACGTGATCGTGGCGGCGCTGGACGGGGACTTTAT

E1-3,6 516 GCAGCAGCCCTTCAAGCAGGTGACGGCGTTGGTGCCCATGGCGGACAAGCTGGACAAGCTGACGGCGGTG E5 516 GCAGCAGCCCTTCAAGCAGGTGACGGCGTTGGTGCCCATGGCGGACAAGCTGGACAAGCTGACGGCGGTG E7 516 GCAGCAGCCCTTCAAGCAGGTGACGGCGTTGGTGCCCATGGCGGACAAGCTGGACAAGCTGACGGCGGTG GCAGCAGCCCTTCAAGCAGGTGACGGCGTTGGTGCCCATGGCGGACAAGCTGGACAAGCTGACGGCGGTG GCAGCAGCCCTTCAAGCAGGTGACGGCGTTGGTGCCCATGGCGGACAAGCTGGACAAGCTGACGGCGGTG

TGCATGAAGTGCAAGATGCGCGACGCACCCTTCACCGTCAGAATCTCTCAGGGCACGGACCTGGTCCAGG 586 TGCATGAAGTGCAAGATGCGCGACGCACCCTTCACCGTCAGAATCTCTCAGGGCACGGACCTGGTCCAGG 586 TGCATGAAGTGCAAGATGCGCGACGCACCCTTCACCGTCAGAATCTCTCAGGGCACGGACCTGGTCCAGG 586 TGCATGAAGTGCAAGATGCGCGACGCACCCTTCACCGTCAGAATCTCTCAGGGCACGGACCTGGTCCAGG 586 TGCATGAAGTGCAAGATGCGCGACGCACCCTTCACCGTCAGAATCTCTCAGGGCACGGACCTGGTCCAGG 586 TGCATGAAGTGCAAGATGCGCGACGCACCCTTCACCGTCAGAATCTCTCAGGGCACGGACCTGGTCCAGG

445

445

445

445

445

445

515

515

515

515

515

515

585

585

585

585

585

585

655

655

655

655

655

655

656 TTGGAGGCGCCGAGTCTTACCAGGCGGTGTGTCGTCCCTGTCTCACGGGGTTCAGGATGGCCCAGTACGA

656 TTGGAGGCGCCGAGTCTTACCAGGCGGTGTGTCGTCCCTGTCTCACGGGGTTCAGGATGGCCCAGTACGA

1-3,6 726 GCTGTACGGTCCGCCGCCTCCTCCTCCTGCGCATAATCTACTGGGTGCGCCCGTCGTGTCAGCCGCTCCA 726 GCTGTACGGTCCGCCGCCTCCTCCTCCTGCGCATAATCTACTGGGTGCGCCCGTCGTGTCAGCCGCTCCA 726 GCTGTACGGTCCGCCGCCTCCTCCTCCTGCGCATAATCTACTGGGTGCGCCCGTCGTGTCAGCCGCTCCA

726 GCTGTACGGTCCGCCGCCTCCTCCTCCTGCGCATAATCTACTGGGTGCGCCCGTCGTGTCAGCCGCTCCA 726 GCTGTACGGTCCGCCGCCTCCTCCTCCTCCGCATAATCTACTGGGTGCGCCCATCGTGTCAGCCGCTCCA 726 GCTGTACGGTCCGCCGCCTCCTCCTCCTGCGCATAATCTACTGGGTGCGCCCATCGTGTCAGCCGCTCCA stop annealing position of primer TKspecER7C

Polyadenylylation site 795 795 795 795 795 795

E1-3,6 796 CCTCGTTCTTGTAACATATCTATCCTGTGATGGTGTGTGTGGAACCAATAAAAT--TGTGCGACTTGAAT 863 E5 796 CCTCGTTCTTGTAACATATCTATCCTGTGATGGTTGTGTGGAACCAATAAAAT--TGTGCGACTTGAAT 863 E7 796 CCTCGTTCTTGTAACATATCTATCCTGTGATGGTGTGTGTGGAACCAATAAAAT--TGTGCGACTTGAAT 863 E4 796 CCTCGTTCTTGTAACATATCTATCCTATGATGGTGTTGTGGAACCAATAAAATAATGTGCGACTTGAAT 865 A1 796 CCTCGTTCTTGTAAC--ATCTATCCTGTGATGGTGTGTGTGGAACCAATAAAAFAATTGCGACTTGAAT 863 A2 796 CCTCGTTCTTGTAAC--ATCTATCCTGTGATGGTGTGTGTGGAACCAATAAAAEAATGTGCGACTTGAAT 863 $\longleftarrow$ annealing position of primer TKspecAR3A5A polyA signal ${ }^{\star \star}$
stop

E1-3,6 864 ATGGTTGTACGGGTTTTTTTTTAACAAAAACTAAACTACCGAAACACGAAACACTTGCTCTGAGCGACTT 933 E5 864 ATGGTTGTACGGGTTTTTTTTAACAAAAACTAAACTACCGAAACACGAAACACTTGCTCTGAGCGACTT 933 E7 864 ATGGTTGTACGGGTTTTTTTT-AACAAAAACTAAACTACCGAAACACGAAACACTTGCTCTGAGCGACTT 932 E4 866 ATGGTTGTACGGGTTTTTTTTAACAAAAACTAAACTACCGAAACACGAAACACTTGCTCTGAGCGACTT 935 A2 864 ATGGTTGTACGGGTTTTTTTT-AACAAAAACTAAACTACCGAAACACGAAACACTTGCTCTGAGCGACTT 932

E1-3,6 934 TGCGTCCAATACTTTAAAAAAAACAGGAGATATTAAATATAGTTCAAACGTTTATTGGGATACACACA 1001 E5 934 TGCGTCCAATA-------------GG------AAATATAGTTCAAACGTTTATTGGGATACACACA E7 933 TGCGTCCAATACTTTAAAAAAAACAGGAGATATTAAATATAGTTCAAACGTTTATTGGGATACACACA E4 936 TGCGTCCAATACTTTAAAAAAAACAGGAGATATTAAATATAGTTCAAACGTTTATTGGGATACACACA A1 932 TGCGTCCAATACTTTAAAAAAAA--GGAGATATTAAATATAGTTCAAACGTTTATTGGGATACACACA A2 933 TGCGTCCAATACTTTAAAAAAAA--GGAGATATTAAATATAGTTCAAACGTTTATTGGGATACACACA

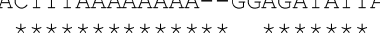

$$
\begin{array}{r}
980 \\
1000 \\
1003 \\
997 \\
998
\end{array}
$$

Fig. 1. Multiple alignment of the partial enlarged TK gene sequences. Only partial sequences around the stop codon are shown. Abbreviations of genotype/variant are the same as those in Table 2. Numbers indicate nucleotide positions. Disagreements of nucleotides are indicated by asterisks. Stop codons are denoted by rectangles. The polyadenylylation site determined by 3' RACE and annealing positions of newly designed primers to distinguish between Asian and European genotypes of KHV are shown using arrows. 
variants, respectively.

Only two variants (A1 and A2) were found in KHVs collected from Asian countries based on one base "T" deletion found in serial " $T$ " residues (No. 877-885) in the enlarged TK gene region (Table 2). These Asian variants differed from European KHV variants in six or seven variable areas and formed an Asian genotype, which can be clearly distinguished from the European genotype of KHV mentioned below. In particular, the C-terminal amino acid residues of the TK gene of the Asian genotype varied greatly by frame-shift compared to the European genotype of KHV (Figs. 1, 2). The reference strain of the Japanese KHV, NRIA-0301, was classified as variant $A 1$ and we could not find any sequence differences among all of the KHV samples collected in Japan. This A1 variant was also found in all other Asian countries; Taiwan, Philippines and Indonesia where KHV was obtained for this study. The other variant of the Asian genotype, A2, was not found in Japan or the Philippines but was present in Indonesia and Taiwan. The presence of two variants in Indonesia and Taiwan suggests either that KHV has invaded these areas at least twice, or less likely, the same subtype differentiation occurred independently after a single KHV invasion in these areas. The latter might be possible, because the deletions or insertions in the same serial nucleotide residues are considered to be easy to occur by slippage of the DNA polymerase (Streisinger et al., 1966; Okada et al., 1972; Streisinger and Owen, 1985; Kunkel, 1990; Kroutil et al., 1996; Sia et al., 1997; Strauss, 1999; Gragg et al., 2002; Kühner et al., 2007). In Indonesia, the A2 variant was found only in the west and north of Sumatra Island, whereas all KHV found in east Sumatra was A1. This differential localization of KHV variants in Sumatra Island is in agreement with epidemiological information that the Toba Lake in North Sumatra Province was contaminated with KHV due to the movement of infected juvenile common carp from West Sumatra Province (Sunarto 2004*4;

\footnotetext{
EURO 1 MAMLELVIGPMFAGKSTESCRRLERLSYSGRRCIAVKHAIDQRYTEESKV 50 ASIA 1 MAMLELVIGPMFAGKSTESCRRLERLSYSGRRCIAVKHAIDQRYTEESKV 50

EURO 51 AMHSGATYPAISAGYLYEVMQRLEEYDAVAVDEGQFFPDLYEGVVQLLTA 100 ASIA 51 AMHSGATYPAISAGYLYEVMQRLEEYDAVAVDEGQFFPDLYEGVVQLLTA 100

EURO 101 GKYVIVAALDGDFMQQPEKQVTALVPMADKLDKLTAVCMKCKMRDAPFTV 150 ASIA 101 GKYVIVAALDGDFMQQPEKQVTALVPMADKLDKLTAVCMKCKMRDAPFTV 150

EURO 151 RISOGTDLVOVGGAESYQAVCRPCLTGFRMAQYELYGPPPPPPAHNLIGA 200 ASIA 151 RISQGTDLVQVGGAESYQAVCRPCLTGFRMAQYELYGPPPPPPAHNLLGA 200

EURO 201 PVVSAAPRRSCISIL 216

ASIA 201 PIVSAAPPRSCNIYPVMVCVEPIK 224
}

Fig. 2. Alignment of the full-length amino acid sequences deduced from the TK gene of European (EURO) and frame-shifted Asian (ASIA) KHVs. Numbers mean amino acid positions. Disagreements of amino acid residues are indicated by asterisks.

\footnotetext{
*4 Sunarto, A. (2004): Epidemiology, diagnostic and preventive practices for koi herpesvirus (KHV) in Indonesia. Abstract of
}

Yuasa, 2007). The homogeneity of Asian KHVs suggests that invasion of $\mathrm{KHV}$ into these areas occurred more recently and the virus spread rapidly when compared to the case of European KHVs mentioned below. In 2002, a KHV outbreak of imported koi occurred in a farm in Guangdong Province of continental China (Liu et al., 2002). Although it was not clear which country the koi were imported from, the reported sequence of $9 / 5$ fragment of this KHV has a characteristic of Asian genotype (the number of serial "A" residues in this region is six and not seven).

In contrast to the Asian genotype of $\mathrm{KHV}$, there were seven variants (E1 E7) in KHVs from Europe, the USA, and Israel. However, these non Asian KHV were closely related to each other when they were compared one by one, and thus composed one genetic group that we have denoted European genotype. The results suggest that KHV from the USA, which was isolated in 1998 from the fish returned from a regional koi show (Hedrick et al., 2000), originated from Europe, since the same variant (E1) was also found in Europe. The variant from Israel (E6) was not found in Europe. However, this Israeli KHV, which was isolated in 1998, was closely related to the $\mathrm{E} 1$ variant. Hence, it is likely that the Israeli variant also originated from one of the European $\mathrm{KHV}$ variants, corresponding to the epidemiological record that KHV was first diagnosed in 1998 after import of koi carp from Europe (Pokorova et al., 2005). The KHV from the UK, which was isolated from koi imported from Malaysia during quarantine, was clearly classified into the European genotype, suggesting the possibility that the koi were infected with KHV in the UK after importation.

When the European variants were compared one by one, differences were found in only one or two genomic regions, although there were six variable regions in total. In contrast, when an Asian variant was compared with any of the European type KHVs, differences were found in at least six regions. The presence of several $\mathrm{KHV}$ variants in Europe, together with the evidence for the presence of KHV in Europe as early as 1996 (Way et al., 2004 ${ }^{* 3}$ ), suggests that the European genotype of $\mathrm{KHV}$ has been present and has diverged for a longer period in Europe than has been observed in Asia. Therefore, it is most likely that Asian KHVs were not introduced from Europe and conversely, European KHVs did not originate from Asia. Thus, the Asian and European genotypes of KHV are likely to have been independently brought to those regions where they contacted and then caused disease in naive populations of koi and common carp.

Although no intermediate type between the European and Asian genotypes was found in this study,

Tokyo University of Marine Science and Technology workshop 'KHV infection -Present Status and Future Prospects for Prevention- -in Tokyo. 
the insertion of double " $A$ " residues in the enlarged TK gene region of the $\mathrm{E} 4$ variant may indicate some relation to the Asian genotype, which has the same insertion. This double " $A$ " insertion located between the polyadenylylation signal and polyadenylylation site is indispensable to create the new stop codon for the frame-shifted TK gene of the Asian genotype (Fig. 1). This suggests that a possible prototype of KHV had this insertion, which is retained in the E4 variant similar to the Asian genotype while other European variants have lost these residues.

The samples from Europe in this study represent a limited geographic range and additional isolates need to be analyzed. If an intermediate type between the European and Asian genotypes of KHV is found, it would provide a strong indication of the potential origins of the virus. Recently, full genome sequences of two European variants (USA and Israel) of KHV and one Asian variant of KHV were reported (Aoki et al., 2007). A detailed comparison of the genome sequence would present further epidemiological information on KHV.

From the results of the present study, the Asian and European genotypes of KHV can be easily distinguished from each other by typing the frame-shifted region of the TK gene. This will be useful in future epidemiological surveys particularly when the European genotypes of $\mathrm{KHV}$ are introduced to Asian countries or vice versa. We propose an easy PCR method to distinguish the two genotypes using a combination of a common forward primer TKcomF (5'-tgcagcgtctggaggaatac-3'), Europeantype specific reverse primer TKspecER7C (5'-cacaccatcacaggataCatatgt-3'), and Asian-type specific reverse

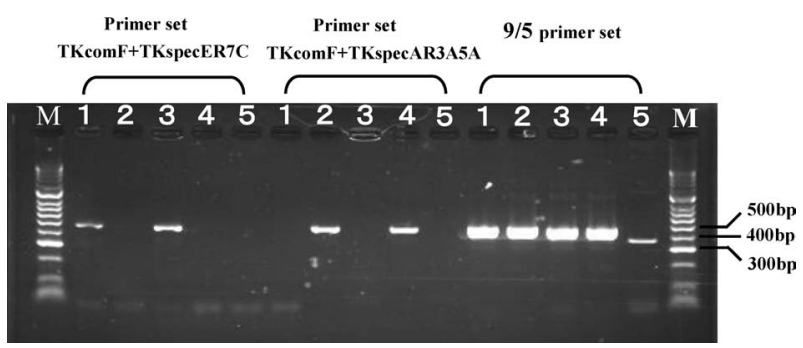

Fig. 3. Agarose gel electrophoresis of PCR products amplified from Asian and European type KHV DNA using the 3 different primer sets. Products were separated on a $1 \%$ agarose gel in TBE buffer. Template DNA was prepared from the supernatant of cell culture infected with E1 KHV (Lane 1), the supernatant of cell culture infected with A1 KHV (Lane 2), the koi tissue affected with $\mathrm{E} 1 \mathrm{KHV}$ (Lane 3), the koi tissue affected with A1 $\mathrm{KHV}$ (Lane 4) and the non-KHV infected common carp tissue as the negative control (Lane 5). Lane $\mathrm{M}$ is the Hyper Ladder II molecular weight marker (BIOLINE, UK). The thermocycling profile was the same as that of 9/5 PCR method by Gilad et al. (2002). Each genotype-specific primer set reacted only with the corrresponding genotype of KHV, whereas the $9 / 5$ primer set reacted both genotypes of KHV. primer TKspecAR3A5A (5'-cacaccatcacaggatagaAgAta3') (Fig. 1) under the conditions of a high annealing temperature of $68^{\circ} \mathrm{C}$ and increased cycles (39 times) (Fig. $3)$. Each reverse primer was designed from the frameshifted region of the TK gene and the nucleotides denoted by capital letters in primer sequences indicate intentionally introduced miss match nucleotides to decrease cross reaction. Primer-specific PCR products of 448 or 450 bp are obtained by this method when sufficient KHV DNA is present. The sequence data of each fragment has been deposited in the DDBJ/EMBL/ GenBank Data Libraries under accession no. AB375379-AB375391.

\section{Acknowledgements}

The authors thank Dr. Oren Gilad (University of Pennsylvania, School of Medicine, Philadelphia, PA) for providing the sequence information upstream and downstream of the $9 / 5$ fragment of the KHV genome and Dr. Joselito R. Somga (Fish Health Section, Bureau of Fisheries and Aquatic Resources, Quezon City, Philippines) for providing the imported KHV samples of the Philippines. This study was supported in part by the research project for utilizing advanced technologies in agriculture, forestry and fisheries, funded by the Ministry of Agriculture, Forestry and Fisheries of Japan.

\section{References}

Aoki, T., I. Hirono, K. Kurokawa, H. Fukuda, R. Nahary, A. Eldar, A. J. Davison, T. B. Waltzek, H. Bercovier and R. P. Hedrick (2007): Genome sequences of three koi herpesvirus isolates representing the expanding distribution of an emerging disease threatening koi and common carp worldwide. J. Virol., 81, 5058-5065.

Bercovier, H., Y. Fishman, R. Nahary, S. Sinai, A. Zlotkin, M. Eyngor, O. Gilad, A. Eldar and R. P. Hedrick (2005): Cloning of the koi herpesvirus (KHV) gene encoding thymidine kinase and its use for a highly sensitive PCR based diagnosis. BMC Microbiol., 5, 1-9.

Bretzinger, A., T. Fischer-Scherl, M. Oumouma, R. Hoffmann and U. Truyen (1999) Mass mortalities in koi, Cyprinus carpio, associated with gill and skin disease. Bull. Eur. Ass. Fish Pathol., 19, 182-185.

Gilad, O., S. Yun, K. B. Andree, M. A. Adkison, A. Zlotkin, H. Bercivuer, A. Eldar and R. P. Hedrick (2002): Initial characteristics of koi herpesvirus and development of a polymerase chain reaction assay to detect the virus in koi, Cyprinus carpio koi. Dis. Aquat. Org., 48, 101-108.

Gragg, H., B. D. Harfe and S. Jinks-Robertson (2002): Base composition of mononucleotide runs affects DNA polymerase slippage and removal of frameshift intermediates by mismatch repair in Saccharomyces cerevisiae. Mol. Cell. Biol., 22, 8756-8762.

Gray, W. L., L. Mullis, S. E. LaPatra, J. M. Groff and A. Goodwin (2002): Detection of Koi herpesvirus DNA in tissues of infected fish. J. Fish Dis., 25, 171-178.

Haenen, O. L. M., K. Way, S. M. Bergmann and E. Ariel (2004): The emergence of koi herpesvirus and its significance to European aquaculture. Bull. Eur. Ass. Fish Pathol., 24, 
293-307.

Hedrick, R. P., O. Gilad, S. Yun, J. V. Spangenberg, G. D. Marty, R. W. Nordhausen, M. J. Kebus, H. Bercovier and A. Eldar (2000): A herpesvirus associated with mass mortality of juvenile and adult koi, strain of common carp. J. Aquat. Anim. Health, 12, 44-57.

lida, T. and M. Sano (2005): Koi herpesvirus disease. Virus, 55, 145-152. (In Japanese with English abstract)

Kroutil, L. C., K. Register, K. Bebenek and T. A. Kunkel (1996): Exonucleolytic proofreading during replication of repetitive DNA. Biochemistry, 35, 1046-1053.

Kühner, F., J. Morfill, R. A. Neher, K. Blank and H. E. Gaub (2007): Force-induced DNA slippage. Biophys. J., 92, 2491-2497.

Kunkel, T. A. (1990): Misalignment-mediated DNA synthesis errors. Biochemistry, 29, 8003-8011.

Liu, H., X. Shi, L. Gao and Y. Jiang (2002): Study on the aetiology of koi epizootic disease using the method of nestedpolymerase chain reaction assay (nested-PCR). $J$. Huazhong Agricult. Univ., 21, 414-418. (In Chinese with English abstract)

Morzunov, S. P., J. R. Winton and S. T. Nichol (1995): The complete genome structure and phylogenetic relationship of infectious hematopoietic necrosis virus. Virus Res., 38, 175-192.

Neukirch, M. and U. Kunz (2001): Isolation and preliminary characterization of several viruses from koi (Cyprinus carpio) suffering gill necrosis and mortality. Bull. Eur. Ass. Fish Pathol., 21, 125-135.

Okada, Y., G. Streisinger, J. (E.) Owen, J. Newton, A. Tsugita and M. Inouye (1972): Molecular basis of a mutational hot spot in the lysozyme gene of bacteriophage T4. Nature, 236, 338-341.

Perelberg, A., M. Smirnov, M. Hutran, A. Diamant, Y. Bejerano and M. Kotler (2003): Epidemiological description of a new viral disease affecting cultured Cyprinus carpio in Israel. Israeli J. Aquat.-Bamidgeh, 55, 5-12.

Pokorova, D., T. Vesely, V. Piackova, S. Reschova and J. Hulova (2005): Current knowledge on koi herpesvirus (KHV): a review. Vet. Med.-Czech, 50, 139-147.

Ronen, A., A. Perelberg, J. Abramowitz, M. Hutoran, S. Tinman,
I. Bejerano, M. Steinitz and M. Kotler (2003): Efficient vaccine against the virus causing a lethal disease in cultured Cyprinus carpio. Vaccine, 21, 4677-4684.

Sano, M., T. Ito, J. Kurita, T. Yanai, N. Watanabe, S. Miwa and T. lida (2004): First detection of koi herpesvirus in cultured common carp Cyprinus carpio in Japan. Fish Pathol., 39, $165-167$.

Sano, M., T. Ito, J. Kurita, S. Miwa and T. lida (2005): Diagnosis of koi herpesvirus (KHV) disease in Japan. Bull. Fish. Res. Agen., Supplement No. 2, 59-64.

Sia, E. A., R. J. Kokoska, M. Dominska, P. Greenwell and T. D. Petes (1997): Microsatellite instability in yeast: Dependence on repeat unit size and DNA mismatch repair genes. Mol. Cell. Biol., 17, 2851-2858.

Strauss, B. S. (1999): Frameshift mutation, microsatellites and mismatch repair. Mutat. Res., 437, 195-203.

Streisinger, G. and J. (E.) Owen (1985): Mechanisms of spontaneous and induced frameshift mutation in bacteriophage T4. Genetics, 109, 633-659.

Streisinger, G., Y., Okada, J. Emrich, J. Newton, A. Tsugita, E. Terzaghi and M. Inouye (1966): Frameshift mutations and the genetic code. Cold Spring Harbor Symp. Quant. Biol., 31, 77-84.

Sunarto, A., A. Rukyani and T. Itami (2005): Indonesian experience on the outbreak of koi herpesvirus in koi and carp (Cyprinus carpio). Bull. Fish. Res. Agen., Supplement No. 2, 15-21.

Tu, C., M.-C. Weng, J.-R. Shiau and S.-Y. Lin (2004): Detection of koi herpesvirus in koi Cyprinus carpio in Taiwan. Fish Pathol., 39, 109-110.

Waltzek, T. B., G. O. Kelley, D. M. Stone, K. Way, L. Hanson, H. Fukuda, I. Hirono, T. Aoki, A. J, Davison and R. P. Hedrick (2005): Koi herpesvirus represents a third cyprinid herpesvirus (CyHV-3) in the family Herpesviridae. J. Gen. Virol., 86, $1659-1667$.

Yuasa, K. (2004): Koi herpesvirus (KHV) disease occurred in Indonesia. Ornamental Fish Medicine, 4, 13-16. (In Japanese)

Yuasa, K. (2007): The latest information on koi herpesvirus disease. Ornamental Fish Medicine, 5\&6, 7-11. (In Japanese) 